\title{
Central Venous Access Catheter
}

National Cancer Institute

\section{Source}

National Cancer Institute. Central Venous Access Catheter. NCI Thesaurus. Code

C17612.

A venous catheter positioned with the tip in the proximal third of the superior vena cava, the right atrium, or the inferior vena cava. 